\title{
The Sports Concussion Note: Should SCAT become SCOAT?
}

\author{
Jon Patricios ${ }^{1}$, Robert Collins ${ }^{1}$, Andrew Branfield ${ }^{2}$, Craig Roberts ${ }^{3}$, Ryan Kohler ${ }^{4}$ \\ ${ }^{1}$ Sports Concussion South Africa, Johannesburg, South Africa and the Section of Sports \\ Medicine, Faculty of Health Sciences, University of Pretoria, Pretoria, South Africa \\ ${ }^{2}$ Sports Concussion South Africa, Johannesburg, South Africa \\ ${ }^{3}$ South African Rugby Union, Cape Town, South Africa and the Discipline of Sports Science, \\ University of Kwazulu-Natal, South Africa \\ ${ }^{4}$ Sports Medicine Department, Australian Institute of Sport, Australian Sports Commission, \\ Canberra, Australia \\ Correspondence to: \\ Dr Jon Patricios \\ Sports Concussion South Africa \\ PO Box 1267 \\ Parklands \\ 2121 \\ South Africa \\ Tel. +27 118839000 \\ Fax. +27 112825165 \\ Email jpat@mweb.co.za
}

\section{Key words}

sports concussion, SCAT2

Word count : 2882 


\section{ABSTRACT}

Sports concussion research and clinical guidelines have evolved rapidly. The most recent concussion consensus statement and guidelines (Zurich, 2008) provided clinicians with the SCAT2 as a clinical template for the assessment of acute concussion. For the subsequent serial examinations required for the complete assessment of the concussed athlete, SCAT2 may be inadequate. This paper describes the experience and suggestions of South African sports physicians in evolving a more comprehensive clinical evaluation tool and record of patient care, the Sports Concussion Office Assessment Tool (SCOAT).

\section{INTRODUCTION}

Evaluation of the concussed athlete requires a multifaceted approach.[1-6]The Sports Concussion Assessment Tool version 2 (SCAT2) [2] was designed as a practical assessment tool pertaining particularly to the clinical evaluation of the concussed athlete as discussed at the Zurich Consensus Meeting (2008). SCAT2 has become an international template for clinicians, freely accessible and widely distributed, as requested by the authors, in its original format.

Several versions of concussion symptom scales and assessment tools exist, most based on expert opinion rather than rigorous scientific appraisal, with a recent critical analysis showing no clear supremacy of one tool over another.[7] Important changes in concussion categorisation and recommendations between the Prague[4] and Zurich[2] consensus meetings are an indication that concussion management guidelines are a work in progress. Moreover, groups other than ours have recently suggested modifications to clinical concussion evaluation protocol.[8,9]

South African sports physicians have implemented the use of SCAT2 at international, provincial, club, school and recreational levels in both team and individual sports between 2009 and 2011, the document being made available on local injury-information websites www.sportsconcussion.co.za and www.boksmart.com. This review summarises our groups' combined experience of the SCAT2 particularly regarding how well it fulfils the aims of the Zurich consensus paper and its effectiveness as a clinical evaluation tool. We suggest further refining the document to more effectively monitor the clinical resolution of the concussed athlete in the office environment. An additional clinical tool, the Sports Concussion Office Assessment Tool or SCOAT has been developed which we feel excludes those aspects of the SCAT2 only relevant to the most acute phase of concussion, duplicates the parts relevant in ongoing serial assessments, incorporates more features of significance to the concussed athlete and simplifies the scoring system. 


\section{DEFINITION}

The definition of concussion[2] is necessarily broad with differences in mechanism of injury and the area of the brain involved resulting in a wide spectrum of possible clinical symptoms. SCAT2 makes no provision for the mechanism of injury to be recorded, an aspect that may be of relevance particularly in researching concussion. It is noted that the initial clinical presentation of concussion may mirror that of more serious intra-cranial pathology and the danger signs are listed on the back page of the SCAT2 in a less prominent position than they might need to be. The Zurich definition notes that concussion usually follows a sequential resolution and hence any clinical evaluation tool should be able to monitor progress over time.

\section{CLINICAL EVALUATION}

SCAT2 provides a list of 22 common symptoms associated with concussion scored on a graded scale (0-6) and is of the more inclusive of self-reported symptom scales (SRS). $[1-4,10,11]$ In our experience, symptoms often present in the clusters mentioned in the Zurich consensus statement, reflecting physical, cognitive, emotional or sleep disturbances and it may therefore be useful to group these symptoms. Moreover, of importance to the clinician is the trend in resolution of these symptoms and it would be useful to have a way of, in the same table, recording symptom scores on successive visits. Interestingly, no specific space is allocated to recording whether amnesia, retrograde or anterograde, is present, a symptom often afforded prognostic significance in the concussed athlete.[11-13] The space afforded to the Glasgow Coma Scale and Maddock's Questions reflect the SCAT2's primary focus on fieldside care but are of little significance in follow-up consultations.

The Zurich consensus paper affords a significant amount of discussion to the relevance of modifying factors - additional factors that may influence presentation, recovery and prognosis. Despite this, no reference is made to such factors in the SCAT2, possibly because their influence is difficult to quantify. Nevertheless we feel that, particularly where rapid or same-day return to play may be contemplated (itself highly contentious and rejected in the most recent American College of Sports Medicine guidelines) [14-16] the presence of such modifying factors warrants recording.

Postural stability is a useful measure of clinical progression and the Balance Error Scoring System (BESS) $[17,18]$ in particular, is validated and useful both acutely and in follow-up and is easy to implement during office evaluation.[17-22] 


\section{OVERALL SCORING SYSTEM}

The aim of scoring a patient's assessment is to monitor progress towards clinical resolution. Our first comment is that, despite scoring the severity of symptoms on a range from 0 (absent) to 6 (most severe), the final SCAT2 scoring only considers the total number of symptoms (out of 22) and not their weighting in terms of severity. Aggregating and incorporating the symptom severity scores in the total may better reflect both severity and clinical trend.

The SCAT2 uses a somewhat "mixed" scoring system in that in some aspects of the evaluation such as symptom analysis points are allocated for "negative" findings (i.e. symptoms are present), whereas for other tasks including the aspects of the Standard Assessment of Concussion (SAC) and co-ordination tasks, points are allocated for "positive" responses (i.e. correctly completing the tasks). The BESS combines a "positive" and "negative" score by allocating points for errors and then subtracting the error score from a possible maximum of 30 .

A simpler scoring method would be to only allocate points for significant findings i.e. symptoms that are present or mistakes made during tasks. The ideal score would then be zero.

\section{GLASGOW COMA SCALE}

Similar to other authors[7] we question the relevance of the Glasgow Coma Scale (GCS), a tool developed in the early 1970 s by Jennett and Teasdale specifically for severe head trauma within 6 hours of injury,[23] in a measurement designed to assess a form of mild traumatic head injury. While acknowledging the GCS as alerting to possible structural injury, an athlete scoring anything less than a GCS of 14 is unlikely to be able to complete the SCAT2. Moreover, the athlete presenting for follow-up assessments in the clinician's office is unlikely to have a GCS below 15, hence the GCS is omitted from our Sports Concussion Office Assessment Tool.

\section{INSTRUCTIONS}

The SCAT 2 carefully and meticulously details and integrates instructions to clinicians as to how to use the tool. These instructions should only be necessary in the initial phases of using the instrument, take up considerable space and could perhaps more efficiently be included on a separate page.

\section{THE SCOAT}

The Sports Concussion Office Assessment Tool (SCOAT) is a clinical evaluation tool designed to:

- Be used serially by clinicians in a consulting room environment

- Evolve from the SCAT2 by retaining the important features relevant to acute, subacute and subsequent presentations 
- Exclude those aspects of SCAT2 only relevant to the fieldside assessment of concussion

- Follow the Zurich consensus guidelines as closely as possible

- Simplify the scoring system to one that tends towards zero before returning to play

- Aid as a tool for further research

Since the beginning of 2009, as group of South African sports physicians we have been involved with the assessment of concussed athletes from international to school level. We have used the SCAT2 as our standard assessment template in assessing over 500 athletes mostly rugby union players at international, Super Rugby, provincial, club and school level, but including participants in soccer, field hockey, cricket, horse riding, cycling and moto-x. The SCOAT represents a tool we feel more comprehensively evaluates the concussed athlete in an office environment while remaining true to the Zurich guidelines.

\section{FORMAT}

It is assumed that clinicians using the SCOAT will be familiar with the SCAT2 and the instructions provided therein. Where instructions are necessary for the patient these are retained. In any case the use of the SCOAT is for the most part self-explanatory; hence many of the instructions in SCAT2 are omitted creating more space for the recording of the clinical evaluation.

\section{EPIDEMIOLOGICAL DATA}

These are expanded upon from the SCAT2 with the addition of "position played" and the "mechanism of injury" both of which may be relevant in epidemiological data capture. Loss of consciousness (LOC) and, in particular, amnesia are specifically recorded. Rather than an emphasis on the presence and duration of LOC which heavily influenced previous grading scales and return to play (RTP), amnesia is now acknowledged as the more significant acute marker of injury severity and duration.[12,13,24] Moreover, in our experience, how soon the player is removed from the field after the injury may influence the potential for aggravating trauma and hence recovery and is therefore also noted. Finally in this section a record of whether protective equipment was used may be useful for retrospective statistical analysis of the influence of such equipment on both concussion and associated injuries (recorded in another section of the SCOAT).

\section{"RED FLAGS"}

As a reminder to clinicians that more serious intra-cranial pathology may mimic concussion particularly in the early stages of presentation,[22] those symptoms that may indicate a need for neuroimaging and neurosurgical referral are afforded more prominence than in the SCAT2. 


\section{COMPLIANCE RECORD}

We have found that patient recovery is often influenced by compliance to the counsel given. For example:

- Was a concussed child kept from school?

- $\quad$ Did he/she avoid unnecessary forms of cognitive stress (computer games; texting etc.)?

- Were all forms of physical exertion avoided?

Failure to adequately comply may explain slower progress and is therefore recorded; similarly, claims of good compliance in the face of a failure to clinically improve may be a poor prognostic sign.

\section{MODIFYING FACTORS}

The Zurich consensus paper[2] places significant emphasis on the potential influence of modifying factors on the nature of presentation and recovery from concussion. These associations are noted to possibly influence predisposition to injury, prognosis and recovery. In our experience, it is most beneficial to document such features as a qualitative record that may significantly influence player management, in particular the timing of RTP.

\section{SYMPTOM ANALYSIS}

One of the most influential determinants of clinical progress is the progression of symptom resolution. Previous authors have pointed out that symptom scales used for concussion have not been systematically validated for reliability, sensitivity and specificity[7,10] and a good case is made for reducing the number of symptoms assessed to 12 as in the Concussion Symptom Inventory.[25] For the purpose of comparison with fieldside SCAT analysis performed more acutely, the SCOAT retains the same 22 symptoms as SCAT2 and the 7-point Likert scale form common to most concussion symptom scales[10] but divides them into clusters, a method used in at least one other previously described assessment form.[26] The advantage of this is that the clinician is able to more efficiently monitor trends in both presentation (i.e. are the manifestations of injury more physical, cognitive, emotional or sleep-related?) and resolution. The assessor is encouraged to monitor the trend by marking scores from successive consultations on the same table using different coloured markers. Hence the symptom-score on the day of injury (transferred from the SCAT2 or recalled retrospectively) is initially marked in one colour and scores from all subsequent consultations in others, ideally serially trending towards a score of 0/6 for each.

The changing of symptoms with exercise or cognitive stress is recorded unchanged whilst a third party record of whether behaviour has changed is still included but elaborated on by providing the option "Somewhat Different" and a space for a short qualitative description of the changes. 


\section{VERBAL COGNITIVE ASSESSMENT}

The clinical cognitive assessment (cognitive assessment, immediate memory, concentration and delayed recall) as described and validated in the Standardized Assessment Concussion (SAC) [27-29] is left almost unchanged from SCAT2. Of note however, is that SCOAT uses a modified scoring system where incorrect answers are awarded 1 point. The purpose of this is to again create a "norm" of zero mistakes, the aggregate score in the recovering patient trending towards zero.

\section{BALANCE ASSESSMENT}

The validated Balance Error Scoring System (BESS) [17] is left unchanged as is the awarding of a point for each error scored, again aiming for a "normal" score of zero.

\section{EXAMINATION}

As the SCOAT is designed as a clinical evaluation form, space is afforded to clinical examination findings both general and neurological. Associated injuries in particular neck and maxillofacial injuries are recorded both as part of the clinical assessment and because of their possible association with concussion as part of the same mechanism of injury. Again a score of 1 point for any adverse finding is awarded as an acknowledgement that players should have a normal neurological examination before returning to sport.

\section{COMPUTERISED COGNITIVE SCREENING}

The trend in concussion evaluation is towards thorough and serial clinical assessments.[2] However, the emergence of computerised cognitive test batteries has provided an additional validated tool enabling the clinician to evaluate changes in brain function particularly where a preinjury baseline score is available.[30-35] Studies suggest a greater sensitivity of computerised cognitive testing batteries to concussion resolution when compared to symptom evaluation.[36] The SCOAT provides space for such serial results to be documented and integrated into the final return-to-play decision.

\section{NEUROIMAGING}

Because the manifestations of concussion are largely functional rather than structural, brain scans (CT or MRI) have specific indications.[37] In those cases where imaging is warranted, the indication, type of scan and result is recorded on the SCOAT. Newer, emerging forms of imaging technology such as proton magnetic resonance spectroscopy (H-MRS) [38] and diffusion tensor imaging (DTI) [39] are not readily available, affordable or accessible yet in South Africa but may warrant inclusion later should they show important clinical information. 


\section{SCORING SYSTEM}

One of the significant changes between the SCOAT and SCAT2 templates is the scoring system. As described earlier, whereas the SCAT2 awards points for both adverse findings (e.g. symptoms and balance errors) as well as correctly completing tasks (e.g. the components of the SAC), the SCOAT simplifies the scoring by only awarding points for adverse or incorrectly completed tasks The importance of the symptom analysis is also emphasised in the SCOAT by weighting it according to the sum of the points awarded for each symptom and not just the number of positive symptoms.

The Maddock's and GCS scores, being significant only in the most acute stages, are left out. Instead additional scores are symptoms (weighted for severity out of a possible total of 132) and physical findings (haemodynamic, associated injuries and neurological).

The maximum aggregate score is 200 and before returning to play the patient should ideally score zero meaning there are no symptoms, the examination is normal and the verbal cognitive, co-ordination and balance tasks were flawlessly completed. In a fully recovered patient this may still not be possible where baseline measures of these parameters are not perfect, but we feel that zero provides a benchmark towards which all recovering concussed athletes should trend.

\section{RECOMMENDED MANAGEMENT}

No space is afforded on the SCAT2 for recording how the concussed player is managed. The SCOAT provides a record of medications prescribed, referrals to colleagues and paperwork dispensed.

\section{CHECKLIST}

The final block is a checklist of those factors most highlighted in the consensus statements [1-5] and essential for ensuring that the athlete is recovered before returning to sport namely that they are asymptomatic, have a normal examination, the computerised cognitive screen has normalised and the return-to-play exercise protocol has been followed whilst acknowledging the influence of modifying factors.

Table 1 summarises the key aspects of the SCOAT especially where these differ from the SCAT2, whilst the PDF version of the document is available at http://www.sportsconcussion.co.za/Documents/SCOAT.pdf 


\begin{tabular}{|l|}
\hline \multicolumn{1}{|c|}{ Table 1. Key Aspects of SCOAT } \\
\hline More detailed epidemiological data \\
\hline Records mechanism of injury \\
\hline Highlights clinical "Red Flags" \\
\hline Records management advice and compliance \\
\hline Documents potential modifying factors for recovery and prognosis \\
\hline Facilitates documentation of serial symptom analysis \\
\hline Weighted scoring for symptoms according to severity on Likert scale \\
\hline Documents examination findings - general, neurological and associated injuries \\
\hline Documents computerised cognitive scores for integration with clinical findings \\
\hline Documents management guidelines and referrals \\
\hline $\begin{array}{l}\text { Simplified scoring system - patient scores zero for normal findings; } \\
\text { recovering patient's aggregate score trends to zero }\end{array}$ \\
\hline Final checklist before allowing return-to-play \\
\hline
\end{tabular}

\section{VALIDITY}

As with all such concussion assessment tools, the SCOAT has evolved from clinical experience and expert opinion, preceding rather than following a process of scientific validation. Nevertheless, our aim in developing this clinical aide is to include all aspects that both science and our experience suggest are relevant to the evaluation and monitoring of the concussed athlete. Moreover, baseline assessment of athlete symptom scales, balance evaluations and cognitive ability has been shown to further improve the accuracy of post-concussion clinical evaluations and should be performed with the SCOAT.[7,40-42]

\section{CONCLUSION}

The SCAT2 represents the best attempt yet at converting the principles of the international concussion consensus meetings into a practical clinical tool and is acknowledged by its authors as a work in progress that will be re-evaluated before December 2012. Having worked with SCAT2 for 2 years, the SCOAT embodies the ideas of South African sports physicians in an attempt to evolve a tool that encompasses the essential tenets of concussion consensus, embodies every relevant aspect of clinical care and is more pragmatic in the office environment, whilst acknowledging the benefits of baseline testing and the need for scientifically validating such an instrument. It is intended that this instrument will further contribute to the evolution of international sports concussion clinical protocol.

\section{Competing interests: none}




\section{References}

1 Aubry M, Cantu R, Dvorak J et al. Summary and agreement statement of the First International Conference on Concussion in Sport, Vienna 2001. Recommendations for the improvement of safety and health of athletes who may suffer concussive injuries. Br J Sports Med 2002;36(1):610.

2 McCrory P, Meeuwisse W, Johnston K et al. Consensus statement on Concussion in Sport--the 3rd International Conference on Concussion in Sport held in Zurich, November 2008. J Sci Med Sport 2009;12(3):340-351.

3 Guskiewicz KM, Bruce SL, Cantu RC et al. Research based recommendations on management of sport related concussion: summary of the National Athletic Trainers' Association position statement. Br J Sports Med 2006;40(1):6-10.

4 McCrory P, Johnston K, Meeuwisse W, et al. Summary and agreement statement of the 2nd International Conference on Concussion in Sport, Prague 2004. Br J Sports Med 2005;39(4):196204.

5 Herring S, Bergfield J, Boland A, et al. Concussion (mild traumatic brain injury) and the team physician: a consensus statement. Med Sci Sports Exerc 2006 ;38(2):395-399.

6 Notebaert AJ, Guskiewicz KM. Current Trends in Athletic Training Practices for Concussion Assessment and Management. J Athl Train 2005;40(4):320-335.

7 Eckner JT, Kutcher JS. Concussion symptom scales and sideline assessment tools: a critical literature update. Curr Sports Med Rep 2010 Jan-Feb;9(1):8-15.

8 NFL Sideline Concussion Assessment Tool. Available at: http://nflhealthandsafety.files.wordpress.com/2011/01/nfl-concussion-tool-post-injury.pdf. Accessed 06/19, 2011.

9 Furman J, Mucha A, Collins M. UPMC Sports Concussion Program 2010 Physical : Vestibular/Balance exam. Available at: http://www.beyondprinting.com/sportsmed/PDF/fri/MGT FRI 04\%20\%5BCompatibility\%20Mode \%5D.pdf. Accessed 06/19, 2011.

10 Alla S, Sullivan SJ, Hale L, McCrory P. Self-report scales/checklists for the measurement of concussion symptoms: a systematic review. Br J Sports Med 2009;43(Suppl 1):3-12.

11 McCrory PR, Johnston KM. Acute clinical symptoms of concussion: assessing prognostic significance. Sports Med 2002;30(8):43-47.

12 Reddy CC, Collins MW. Sports concussion: management and predictors of outcome. Curr Sports Med Rep 2009;8(1):10-15.

13 Cantu RC. Posttraumatic retrograde and anterograde amnesia: pathophysiology and implications in grading and safe return to play. J Athl Train 2001;36:244-248.

14 Putukian M, Aubry M, McCrory P. Return to play after sports concussion in elite and non-elite athletes? Br J Sports Med 2009;43(Suppl 1):28-31. 
15 Guskiewicz KM, McCrea M, Marshall SW et al. Cumulative effects associated with recurrent concussion in collegiate football players: the NCAA Concussion Study. JAMA 2003;290(19):25492555.

16 Lovell M, Collins M, Bradley J. Return to play following sports-related concussion. Clin Sports Med $2004 ; 23(3): 421-441$.

17 Guskiewicz KM. Postural stability assessment following concussion: one piece of the puzzle. Clin J Sport Med 2001;11(3):182-189.

18 Guskiewicz KM, Ross SE, Marshall SW. Postural stability and neuropsychological deficits after concussion in collegiate athletes. J Athl Train 2001;36:263-273.

19 McCrea M, Guskiewicz KM, Marshall SW et al. Acute effects and recovery time following concussion in collegiate football players: the NCAA Concussion Study. JAMA 2003; 290(19):2556-2563.

20 Peterson CL, Ferrara MS, Mrazik M,et al. Evaluation of neuropsychological domain scores and postural stability following cerebral concussion in sports. Clin J Sport Med 2003;13(4):230237.

21 Riemann BL, Guskiewicz KM. Relationship between clinical and force plate measures of postural stability. J Sport Rehabil 1999;8:71-82.

22 Riemann BL, Guskiewicz KM. Effects of mild head injury on postural stability as measured through clinical balance testing. J Athl Train 2000;35:19-25.

23 Fischer J, Mathieson C. The history of the Glasgow Coma Scale: implications for practice. Crit Care Nurs 2001;23(4):52-58.

24 Erlanger D, Kaushik T, Cantu R et al. Symptom-based assessment of the severity of a concussion. J Neurosurg 2003 ;98(3):477-484.

25 Randolph C, Millis S, Barr WB et al. Concussion symptom inventory: an empirically derived scale for monitoring resolution of symptoms following sport-related concussion. Arch Clin Neuropsychol 2009 ;24(3):219-229.

26 Gioia GA, Collins M. Available at: http://www.cdc.gov/concussion/headsup/pdf/ACE care plan school version a.pdf. Accessed 06/16, 2011.

27 McCrea M, Kelly JP, Randolph C et al. Standardized assessment of concussion (SAC): on-site mental status evaluation of the athlete. J Head Trauma Rehabil 1998 ;13(2):27-35.

28 McCrea M, Kelly JP, Kluge J, Ackley B et al. Standardized assessment of concussion in football players. Neurology 1997;48(3):586-588.

29 Grubenhoff JA, Kirkwood M, Gao D et al. Evaluation of the standardized assessment of concussion in a pediatric emergency department. Pediatrics 2010;126(4):688-695.

30 Collie A, Darby D, Maruff P. Computerised cognitive assessment of athletes with sports related head injury. Br J Sports Med 2001;35(5):297-302. 
31 Collie A, Maruff P. Computerised neuropsychological testing. Br J Sports Med 2003;37(1):2-3.

32 Collie A, Maruff $P$, McStephen $M$ et al. Psychometric issues associated with computerised neuropsychological assessment of concussed athletes. Br J Sports Med 2003;37(6):556-559.

33 Collins MW, Grindel SH, Lovell MR et al. Relationship between concussion and neuropsychological performance in college football players. JAMA $1999 ; \mathbf{2 8 2 ( 1 0 ) : 9 6 4 - 9 7 0 . ~}$

34 Lovell MR. The relevance of neuropsychologic testing for sports-related head injuries. Curr Sports Med Rep 2002;1(1):7-11.

35 Lovell MR, Collins MW. Neuropsychological assessment of the college football player. $J$ Head Trauma Rehabil 1998;13(2):9-26.

36 Makdissi M, Darby D, Maruff $P$ et al. Natural history of concussion in sport: markers of severity and implications for management. Am J Sports Med 2010;38(3):464-471.

3) Ellemberg D, Henry LC, Macciocchi SN et al. Advances in sport concussion assessment: from behavioral to brain imaging measures. J Neurotrauma 2009;26(12):2365-2382.

38 Vagnozzi R, Signoretti S, Cristofori L et al. Assessment of metabolic brain damage and recovery following mild traumatic brain injury: a multicentre, proton magnetic resonance spectroscopic study in concussed patients. Brain 2010;133(11):3232-3242.

39 Maruta J, Lee SW, Jacobs EF et al. A unified science of concussion. Ann N Y Acad Sci 2010;1208:58-66.

40 Shehata N, Wiley JP, Richea S et al. Sport concussion assessment tool: baseline values for varsity collision sport athletes. Br J Sports Med $2009 ; 43(10): 730-734$.

41 Schneiders AG, Sullivan SJ, Gray AR et al. Normative values for three clinical measures of motor performance used in the neurological assessment of sports concussion. J Sci Med Sport 2010;13(2):196-201.

42 Finnoff JT, Peterson VJ, Hollman JH et al. Intrarater and interrater reliability of the Balance Error Scoring System (BESS). PMR 2009 ;1(1):50-54. 